\title{
DISTRIBUTION OF THE MEAN MOTIONS OF PLANETS \\ AND SATELLITES AND THE DEVELOPMENT OF \\ THE SOLAR SYSTEM
}

\author{
H. JEHLE \\ Center for Theoretical Physics, Department of Physics and Astronomy, University of Maryland, \\ College Park, Md. 20742, U.S.A., and Physics Department, George Washington University, \\ Washington, D.C. 20006, U.S.A.
}

\begin{abstract}
In this paper the point of view is taken that the distribution of orbital elements in the solar system should be discussed first on a purely gravitational basis, i.e. on the basis of a set of particles entirely under gravitational interaction, before hydromagnetic and other effects are taken in consideration too. One might indeed assume that there has been a time in the history of the solar system from when on hydromagnetic and gas laws ceased to play an important role in comparison to gravity. In the epoch since that time the solar system might have developed from a set of a large number of smaller particles into the present solar system by way of transitions which these particles made to preferential orbital elements, and by accretion. Means had been found to handle the development of this set of particles under gravitational interaction, by defining the set appropriately in terms of a statistical distribution. In considering the problem of the evolution of the solar system, such a gravitational approach, which was encouraged by Einstein, seems the reasonable first step.
\end{abstract}

